\title{
Single-Trial Decoding of Scalp EEG under Natural Conditions
}

\author{
Greta Tuckute (D), Sofie Therese Hansen (D), Nicolai Pedersen, Dea Steenstrup, \\ and Lars Kai Hansen
}

Department of Applied Mathematics and Computer Science (DTU Compute), Technical University of Denmark, DK-2800 Kongens Lyngby, Denmark

Correspondence should be addressed to Greta Tuckute; grtu@dtu.dk

Received 21 December 2018; Revised 12 February 2019; Accepted 24 February 2019; Published 17 April 2019

Academic Editor: Reinoud Maex

Copyright (C) 2019 Greta Tuckute et al. This is an open access article distributed under the Creative Commons Attribution License, which permits unrestricted use, distribution, and reproduction in any medium, provided the original work is properly cited.

There is significant current interest in decoding mental states from electroencephalography (EEG) recordings. EEG signals are subject-specific, are sensitive to disturbances, and have a low signal-to-noise ratio, which has been mitigated by the use of laboratory-grade EEG acquisition equipment under highly controlled conditions. In the present study, we investigate single-trial decoding of natural, complex stimuli based on scalp EEG acquired with a portable, 32 dry-electrode sensor system in a typical office setting. We probe generalizability by a leave-one-subject-out cross-validation approach. We demonstrate that support vector machine (SVM) classifiers trained on a relatively small set of denoised (averaged) pseudotrials perform on par with classifiers trained on a large set of noisy single-trial samples. We propose a novel method for computing sensitivity maps of EEGbased SVM classifiers for visualization of EEG signatures exploited by the SVM classifiers. Moreover, we apply an NPAIRS resampling framework for estimation of map uncertainty, and thus show that effect sizes of sensitivity maps for classifiers trained on small samples of denoised data and large samples of noisy data are similar. Finally, we demonstrate that the average pseudotrial classifier can successfully predict the class of single trials from withheld subjects, which allows for fast classifier training, parameter optimization, and unbiased performance evaluation in machine learning approaches for brain decoding.

\section{Introduction}

Decoding of brain activity aims to predict the perceptual and semantic content of neural processing based on activity measured in one or more brain imaging modalities, such as electroencephalography (EEG), magnetoencephalography (MEG), and functional magnetic resonance imaging (fMRI). Decoding studies based on fMRI have matured significantly during the last 15 years $[1,2]$, and human brain activity has been successfully decoded from natural images and movies [3-8].

In case of decoding of scalp EEG, the research area is still progressing, and relatively few studies document detection of brain states in regard to semantic categories (often discrimination between two high-level categories) [9-15]. EEGbased decoding of human brain activity has significant potential due to excellent time resolution and the possibility of real-life acquisition; however, the signal is extremely diverse, subject-specific, sensitive to disturbances, and has a low signal-to-noise ratio; hence, posing a major challenge for both signal processing and machine learning [16].

Due to aforementioned challenges, previous studies have been performed in controlled laboratory settings with highgrade EEG acquisition equipment [9-12, 14, 15]. Visual stimuli paradigms can often not be described as naturalistic, due to (1) repeated presentation of identical experimental trials and (2) iconic views of objects and lack of complexity of semantic context [9-14]. Generalizability of decoding classifier models to novel participants is rare, due to subjectspecific modelling approaches [9, 11-15]. Moreover, a number of participants are occasionally excluded from analysis due to artifacts and low classification accuracy $[13,15]$.

The motivation for the present study is to overcome the highlighted limitations in EEG-based decoding. The current experimental paradigm and decoding work is centered around (1) ecological validity and portability and (2) generalizability. Therefore, we acquired scalp EEG signals 
in a typical office setting using a portable, user-friendly, and wireless EEG Enobio system with 32 dry electrodes. Experimental image stimuli consisted of noniconic views of objects embedded in complex everyday scenes (Figure 1(a)) of 23 different semantic categories from an open image database [17]. All images presented were unique and not repeated for the same participant throughout the experiment (Figure 1(b)), akin to how visual stimuli are experienced in real life. We created classifiers based on singletrial responses as well as generalized category representations by averaging responses of images from the same semantic category.

We acquired data from 15 healthy participants (5 females). We are interested in exploring the limitations of intersubject generalization, i.e., population models; hence, no participants are excluded from analysis. Decoding ability is evaluated in an intersubject design, i.e., in a leave-onesubject-out approach (as opposed to within-subject classification) to probe generalizability across participants [18].

The work in the present study is focused on the binary classification problem between two classes: brain processing of animate and inanimate image stimuli. Kernel methods, e.g., support vector machines (SVMs) are frequently applied for learning of statistical relations between patterns of brain activation and experimental conditions. In classification of EEG data, SVMs have shown good performance in many contexts $[10,12,13,19]$ (see [20] for review).

We adopt a novel methodological approach for computing and evaluating SVM classifiers based on two approaches: (1) single-trial training and single-trial test classification and (2) training on an averaged response of each of the 23 image categories for each subject (corresponding to 23 pseudotrials per subject) and single-trial test classification. Furthermore, we open the black box and visualize which parts of the EEG signature are exploited by the SVM classifiers. In particular, we propose a method for computing sensitivity maps of EEG-based SVM classifiers based on a methodology originally proposed for fMRI [21]. To evaluate effect sizes of sensitivity maps and event-related potential (ERP) difference maps, we use a modified version of an NPAIRS resampling scheme [22]. Lastly, we investigate how the pseudotrial classifier based on averaged category responses compares to the single-trial classifier in terms of prediction accuracy of novel subjects.

\section{Materials and Methods}

2.1. Participants. A total of 15 healthy adults with normal or corrected-to-normal vision (10 males, 5 females, mean age: 25, and age range: 21-30), who gave written informed consent prior to the experiment, were recruited for the study. Participants reported no neurological or mental disorders. Noninvasive experiments on healthy subjects are exempt from ethical committee processing by the Danish law [23].

2.2. Stimuli. Stimuli consisted of 690 images from the Microsoft Common Objects in Context (MS COCO) dataset
[17]. Images were selected from 23 semantic categories, with each category containing 30 images. Of the 23 categories, 10 categories contained animals and the remaining 13 categories contained inanimate items, such as food or man-made objects. Thus, each participant was exposed to 300 animate trials and 390 inanimate trials, resulting in a chance level of $56.5 \%$ for prediction of the larger, inanimate class. For categories and image labels used in the experiment, see Supplementary File 1. All images presented were unique and not repeated for the same participant throughout the experiment. The initial selection criteria were (1) image aspect ratio of $4: 3$, (2) only a single super- and subcategory per image, and (3) minimum 30 images within the category. Furthermore, we ensured that all 690 images had a relatively similar luminance and contrast to avoid the influence of lowlevel image features in the EEG signals. Thus, images within $77 \%$ of the brightness distribution and $87 \%$ of the contrast distribution were selected. Images that were highly distinct from standard MS COCO images were manually excluded (see Appendix A for exclusion criteria). Stimuli were presented using custom Python scripts built on PsychoPy2 software [24].

2.3. Experimental Design. Participants were shown 23 blocks of trials composed of 30 images each. The order of categories and images within the categories was random for each participant. At the beginning of each category, a probe word denoting the category name was displayed for $5 \mathrm{~s}$ followed by the 30 images from the corresponding category. Each image was displayed for $1 \mathrm{~s}$, set against a midgrey background. Interstimulus intervals (ISIs) of variable length were displayed between each image. The ISI length was randomly sampled according to a uniform distribution from a fixed list of ISI values between $1.85 \mathrm{~s}$ and $2.15 \mathrm{~s}$ in $50 \mathrm{~ms}$ intervals, ensuring an average ISI duration of $2 \mathrm{~s}$. To minimize eye movements between trials, the ISI consisted of a white fixation cross superimposed on a midgrey background in the center of the screen (Figure 1(b)).

Subjects viewed images on a computer monitor with a viewing distance of $57 \mathrm{~cm}$. The size of stimuli was $4 \times 3$ degrees of visual angle. Duration of the experiment was $39.3 \mathrm{~min}$, which included five $35 \mathrm{~s}$ breaks interspersed between the 23 blocks. Before the experimental start, participants underwent a familiarization phase with two blocks of reduced length (103s).

2.4. EEG Data Collection. User-friendly, portable EEG equipment, Enobio (Neuroelectrics) with 32 dry-electrode channels, was used for data acquisition. The EEG was electrically referenced using a CMS/DRL ear clip. The system recorded 24 bit EEG data with a sampling rate of $500 \mathrm{~Hz}$, which was transmitted wirelessly using Wi-Fi. LabRecorder was used for recording EEG signals. The lab streaming layer (LSL) was used to connect PsychoPy2 and LabRecorder for unified measurement of time series. The system was implemented on a Lenovo Legion Y520, and all recordings were performed in a normal office setting. 

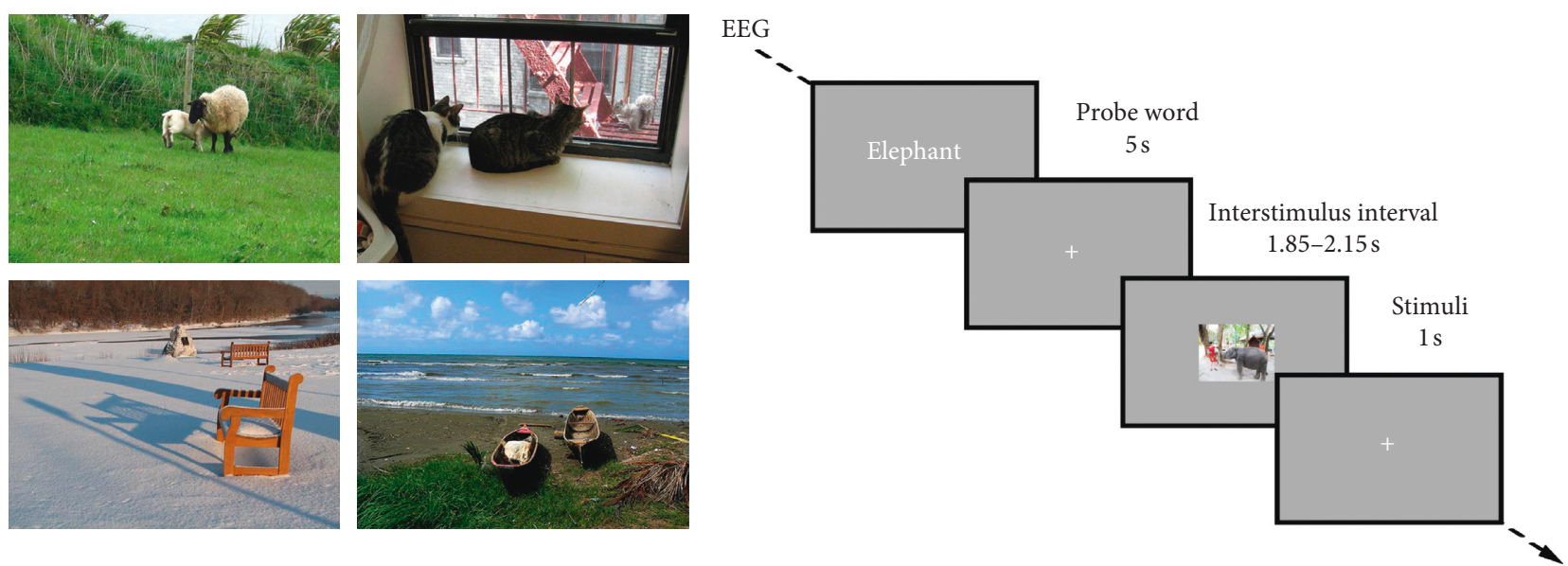

(a)

(b)

Figure 1: (a) Example of the experimental visual stimuli. First row contains animate trials from "sheep" and "cat" categories, and second row contains inanimate trials from "bench" and "boat" categories. (b) Experimental design of the visual stimuli presentation paradigm. The time course of the events is shown. Participants were shown a probe word before each category, and jittered interstimulus intervals consisting of a fixation cross were added between stimulus presentation. The experiment consisted of 690 unique trials in total, 23 categories of 30 trials, ordered randomly (both category- and image-wise) for each subject.

2.5. EEG Preprocessing. Among the 15 recordings, no participants were excluded during data preprocessing, as we would like to generalize our results to a broad range of experimental recordings. Preprocessing of the EEG was done using EEGLAB (sccn.ucsd.edu/eeglab). The EEG signal was bandpass filtered to $1-25 \mathrm{~Hz}$ using finite impulse response filters and downsampled to $100 \mathrm{~Hz}$. Artifact subspace reconstruction (ASR) [25] was applied to reduce nonstationary high-variance noise signals. Temporal trends in the EEG signals were investigated before and after ASR for each subject (Figures S2 and S3). Generally, the time dependencies of the EEG signal were reduced by ASR. Channels that were removed by artifact rejection were interpolated from the remaining channels, and the data were subsequently re-referenced to an average reference. Epochs of $600 \mathrm{~ms}, 100 \mathrm{~ms}$ before and $500 \mathrm{~ms}$ after stimulus onset, similar to [9], were extracted for each trial.

A sampling drift of $100 \mathrm{~ms}$ throughout the entire experiment was observed for all subjects and was corrected for offline.

Since the signal-to-noise ratio varied across trials and participants, all signals were normalized to $z$-score values (i.e., each trial and averaged trials from each participant were transformed so that it had a mean value of 0 and a standard deviation of 1 across time samples and channels).

2.6. Support Vector Machines. Support vector machines (SVMs) were implemented to classify the EEG data into two classes according to animate and inanimate trials. $y_{i} \in\{-1,1\}$ is the identifier of the category, and an observation is defined to be the EEG response in one epoch ([-100, 500] ms w.r.t. stimulus onset). SVMs allow adoption of a nonlinear kernel function to transform input data into a high-dimensional feature space, where it is possible to linearly separate data. The iterative learning process of the SVM will devise an optimal hyperplane with the maximal margin between each class in the high-dimensional feature space. Thus, the maximum-margin hyperplane will form the decision boundary for distinguishing the brain response associated with animate and inanimate data [26].

The SVM classifier is implemented by a nonlinear projection of the observations $\mathbf{x}_{n}$ into a high-dimensional feature space $\mathscr{F}$.

Let $\phi: \mathscr{X} \longrightarrow \mathscr{F}$ be a mapping from the input space $\mathscr{X}$ to $\mathscr{F}$. The weight vector $\mathbf{w}$ can be expressed as a linear combination of the training points $\mathbf{w}=\sum_{n=1}^{N} \alpha_{n} \phi\left(\mathbf{x}_{n}\right)$, and the kernel trick is used to express the discriminant function as

$$
y(\mathbf{x} ; \boldsymbol{\theta})=\alpha^{\mathrm{T}} \mathbf{k}_{x}+b=\sum_{n=1}^{N} \alpha_{n} k\left(\mathbf{x}_{n}, \mathbf{x}\right)+b,
$$

with the model now parametrized by the smaller set of parameter $\boldsymbol{\theta}=\{\boldsymbol{\alpha}, b\}$ [27]. The radial basis function (RBF) kernel allows for implementation of a nonlinear decision boundary in the input space. The RBF kernel $\mathbf{k}_{x}$ holds the elements:

$$
k\left(\mathbf{x}_{n}, \mathbf{x}\right)=\exp \left(-\gamma\left\|\mathbf{x}_{n}-\mathbf{x}\right\|^{2}\right)
$$

where $\gamma$ is a tunable parameter.

Often, it is desirable to allow a few misclassifications in the decision boundary in order to obtain a better generalization error. This trade-off is controlled by a tunable regularization parameter $c$.

Two overall types of SVM classifiers were implemented: (1) single-trial classifier and (2) average category level classifier, denoted as pseudotrial classifier based on the terminology used, for example, in [28]. Both classifiers decode supercategories, animate versus inanimate, and both classify between subjects. The single-trial classifier is trained on 690 trials for each subject included in the training set. The pseudotrial classifier averages the 30 trials within each of the 23 categories for each subject, such that the classifier is 
trained on 23 averaged, pseudotrials for each subject included in the training set, instead of 690 trials.

The performance of the single-trial classifier was estimated using 14 participants as the training set, and the remaining one participant was used as the test set (SVM parameters visualized in Figure S8). Cross-validation was performed on 10 parameter values in ranges $c=[0.05 ; 10]$ and $\gamma=\left[2.5 \times 10^{-7} ; 5 \times 10^{-3}\right]$, thus cross-validating across 100 parameter combinations for each held out subject.

For a debiased estimate of the test accuracy, the singletrial classifier was trained on 13 subjects, with one participant held out for validation and another participant held out for testing, thus leaving out 2 subjects in each iteration. Fifteen classifiers were trained with different subjects held out in each iteration. An optimal parameter set of $c$ and $\gamma$ was estimated using participants 1-7 as validation subjects (mean parameter value), which was used to estimate the test accuracy for subjects $8-15$ and vice versa. Thus, two sets of optimal parameters were found (Figure S10). Cross-validation was performed on 10 parameter values in ranges $c=[0.25 ; 15]$ and $\gamma=\left[5 \times 10^{-7} ; 2.5 \times 10^{-2}\right]$, i.e., 100 combinations.

The pseudotrial classifier was much faster to train and was built using a basic nested leave-one-subject-out crossvalidation loop. In the outer loop, one subject was held out for testing while the remaining 14 subjects entered the inner loop. The inner loop was used to estimate the optimum $c$ and $\gamma$ parameters for the SVM classifier. The performance of the model was calculated based on the test set. Each subject served as a test set once. A permutation test was performed to check for significance. For each left out test subject, the animacy labels were permuted and compared to the predicted labels. This was repeated 1000 times, and the accuracy scores of the permuted sets were compared against the accuracy score of the nonpermuted set. The upper level of performance was estimated by choosing the parameters based on the test set. Cross-validation was performed on 10 parameter values in ranges $c=[0.25 ; 15]$ and $\gamma=\left[5 \times 10^{-7} ; 2.5 \times 10^{-2}\right]$, i.e., 100 combinations.

2.7. Sensitivity Map. To visualize the SVM RBF kernel, an approach proposed by Rasmussen et al. [21] was adapted. The sensitivity map is computed as the derivative of the RBF kernel, c.f. equation (2):

$$
\frac{\partial \alpha^{\mathrm{T}} \mathbf{k}_{x}}{\partial x_{j}}=\sum_{n} \alpha_{n} 2 \gamma\left(x_{n, j}-x_{j}\right) \exp \left(-\gamma\left\|\mathbf{x}_{n}-\mathbf{x}^{2}\right\|\right) .
$$

Pseudocode for computing the sensitivity map across time samples and trials is found in Appendix B. A GitHub toolbox with Python implementation of sensitivity mapping is available: https:/github.com/gretatuckute/DecodingSensitivity Mapping.

2.8. Effect Size Evaluation. The NPAIRS (nonparametric prediction, activation, influence, and reproducibility resampling) framework [22] was implemented to evaluate effect sizes of the SVM sensitivity map and animate/ inanimate ERP differences. The sensitivity map and the
ERP difference map based on all subjects were thus scaled by the average difference of subsampled partitions.

The scaling was calculated based on $S=100$ splits. In each split, two partitions of the dataset were randomly selected without replacement. A partition consisted of 7 subjects, thus achieving two partitions of 7 subjects each (leaving a single, random subject out in each iteration).

For evaluation of the ERP difference map, a difference map was calculated for each partition $\left(\mathbf{M}_{1}\right.$ and $\left.\mathbf{M}_{2}\right)$. Similarly, for evaluation of the sensitivity map, an SVM classifier was trained on each partition, and sensitivity maps were computed for both SVM classifiers (corresponding to $\mathbf{M}_{1}$ and $\mathbf{M}_{2}$ for the ERP difference map evaluation). The sensitivity map for the single-trial SVM classifier was computed based on optimal model parameters, while the sensitivity map of the pseudotrial classifier was based on the mean parameters based on validation sets. The maps from the two partitions were contrasted and squared.

Across time samples $(t=1, \ldots, T)$ and trials $(n=1, \ldots, N)$, an average standard deviation of the average difference between partitions was calculated:

$$
\sigma^{2}=\frac{1}{S T N} \sum_{i, t, n=1}^{S, T, N}\left(\mathbf{M}_{1, t, n}^{i}-\mathbf{M}_{2, t, n}^{i}\right)^{2} .
$$

The full map, $\mathbf{M}_{\text {full }}$ (based on 15 subjects), was then divided by the standard deviation to produce the effect size:

$$
\widehat{\mathbf{M}}=\frac{\mathbf{M}_{\text {full }}}{\sigma} \text {. }
$$

\section{Results}

We classify the recorded EEG using SVM RBF models such that trials are labeled with the high-level category of their presented stimuli, i.e., either animate or inanimate. We first report results using a single-trial classifier followed by a pseudotrial classifier using averaged category responses and then apply the pseudotrial classifier for prediction of singletrial EEG responses. Also, we report effect sizes of ERP difference maps and sensitivity maps for evaluation of both SVM classifiers.

3.1. Event-Related Potential Analysis. After EEG data preprocessing and artifact subspace reconstruction (ASR) (Section 2.5), we confirmed that our visual stimuli presentation elicited a visual evoked response. The ERPs for the trials of animate content and the trials of inanimate content are compared in Figure 2. The grand average ERPs across subjects (thick lines) are shown along with the average animate and inanimate ERPs of each subject. The average scalp map for these two supercategories as well as the difference between them at $310 \mathrm{~ms}$ is displayed in $z$-scored units.

It Figure 2, it is indicated which time samples were significant for the averaged selection of channels. A full map of significant time samples and channels can be seen in Figure S4. The significance level was controlled for multiple comparisons using the conservative Bonferroni correction. 


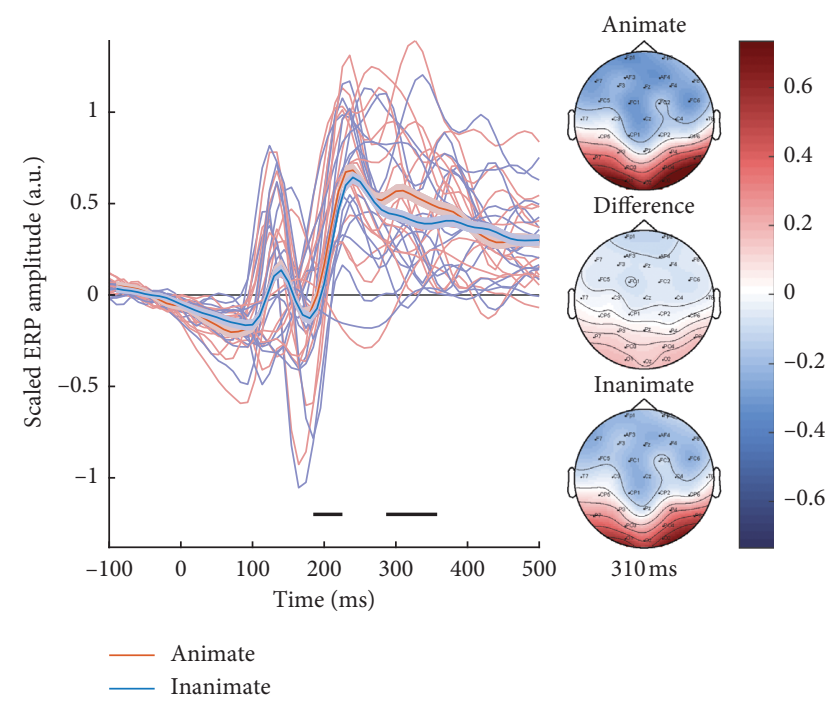

FIgURE 2: Average animate and inanimate ERPs across subjects (thick lines, and with standard errors around the mean) and for each subject (thin lines). ERP analysis was performed on the occipital/parietal channels $\mathrm{O} 1, \mathrm{O} 2, \mathrm{Oz}, \mathrm{PO} 3$, and PO4. The horizontal black lines indicate where the time samples are significant (paired $t$ test corrected for multiple comparisons, $\alpha=0.05 / 60$ time samples). Scalp maps are displayed for the animate/inanimate ERPs and difference thereof at $310 \mathrm{~ms}$.

The animate and inanimate ERPs were most different $310 \mathrm{~ms}$ after stimuli onset. This applied both for the selected channels in Figure 2 and in general, including frontal channels (Figure S4).

Inspection of Figure 2 shows that visual stimuli presentation elicited a negative ERP component at 80-100 ms poststimulus onset followed by a positive deflection at around $140 \mathrm{~ms}$ poststimulus onset. A P300 subcomponent, P3a, was evident around $250 \mathrm{~ms}$ and a P3b component around $300 \mathrm{~ms}$ [29]. It is evident that the P3b component is more prominent for the animate category. The observed temporal ERP dynamics was comparable to prior ERP studies of the temporal dynamics of visual object processing [30].

Mean animate/inanimate ERP responses for each subject separately can be found in Figure S1.

3.2. Support Vector Machines. We sought to determine whether EEG data in our experiment can be automatically classified using SVM models. The Python toolbox scikitlearn [31] was used to implement RBF SVM models.

Specifically, we trained two different types of SVM classifiers, a single-trial and a pseudotrial classifier (averaged category responses), and assessed the classifiers' accuracy on labeling EEG data in a leave-one-subject-out approach.

SVMs are regarded efficient tools for high-dimensional binary as well as nonlinear classification tasks, but their ultimate classification performance depends heavily upon the selection of appropriate parameters of $c$ and $\gamma$ [32]. Parameters for the upper level of performance for the singletrial classifier were found using cross-validation in a leaveone-subject-out approach, resulting in a penalty parameter $c=1.5$ and $\gamma=5 \times 10^{-5}$ based on the optimum mean parameters across test subjects (Figure S9). From Figure S8, it is evident that the optimum parameters were different for each subject, underlining intersubject variability in the EEG responses.

To reduce bias of the performance estimate of the singletrial classifier, parameters were selected based on two validation partitions, resulting in $c=0.5$ and $\gamma=5 \times 10^{-4}$ for the first validation set, and $c=1.5$ and $\gamma=5 \times 10^{-5}$ for the second validation set (Figure S10).

The pseudotrial classifier also showed intersubject variability with respect to the model parameters (see Figures S5-S7). The classifier had an average penalty parameter of $c=7.2$ and an average $\gamma=3.7 \times 10^{-4}$ when based on the validation sets. The average optimum parameters when based on test sets with averaged categories and single trials were in the same range, with $c=4.4$ and $\gamma=3.0 \times 10^{-4}$, and $c=6.7$ and $\gamma=2.2 \times 10^{-4}$, respectively.

Figures 3 and 4 show the SVM classification performances using the two types of classifiers. Based on the leaveone-subject-out classification, we note the large variability of single-subject performance. While different performances are obtained using the single-trial and pseudotrial classifiers on single-trial test sets, the overall accuracies are similar $(p=0.82$, paired $t$-test), with an average of 0.574 and 0.575 , respectively (Figure 4). Thus, the pseudotrial classifier performs on par with the single-trial classifier in the prediction of single-trial test subjects.

A standard error of the mean of 0.01 was found for both the debiased performance measures of the single-trial classifier and for the unbiased single-trial classifier (corrected for the leave-one-subject-out approach [33]).

\subsection{Event-Related Potential Difference Map and Sensitivity} Map. We investigated the raw ERP difference map between animate and inanimate categories, as well as the sensitivity maps for the single-trial and pseudotrial SVM classifiers. The sensitivity map reveals EEG time points and channels that are of relevance to the SVM decoding classifiers (Figure 5).

For map effect size evaluation, we implement an NPAIRS resampling scheme [22]. In this cross-validation framework, the data were split into two partitions of equal size (7 subjects in each partition randomly selected without replacement). This procedure was repeated 100 times to obtain standard errors of the maps for computing effect sizes (Section 2.8).

Figure 5(a) displays the effect sizes of the raw ERP difference map between the animate and inanimate categories, while Figures 5(b) and 5(c) displays effect sizes of sensitivity maps for the single-trial and pseudotrial classifiers, respectively. Scalp maps show the spatial information exploited by the classifiers at different time points.

From inspection of Figure 5, it is evident that occipital and parietal channels (O1, O2, P7, and P8) were relevant for SVM classification at time points comparable to the ERP difference map. Frontal channels (Fp1 and Fp2) were exploited by both SVM classifiers, but to a larger extent by the pseudotrial classifier (Figure 5(c)). Furthermore, the 


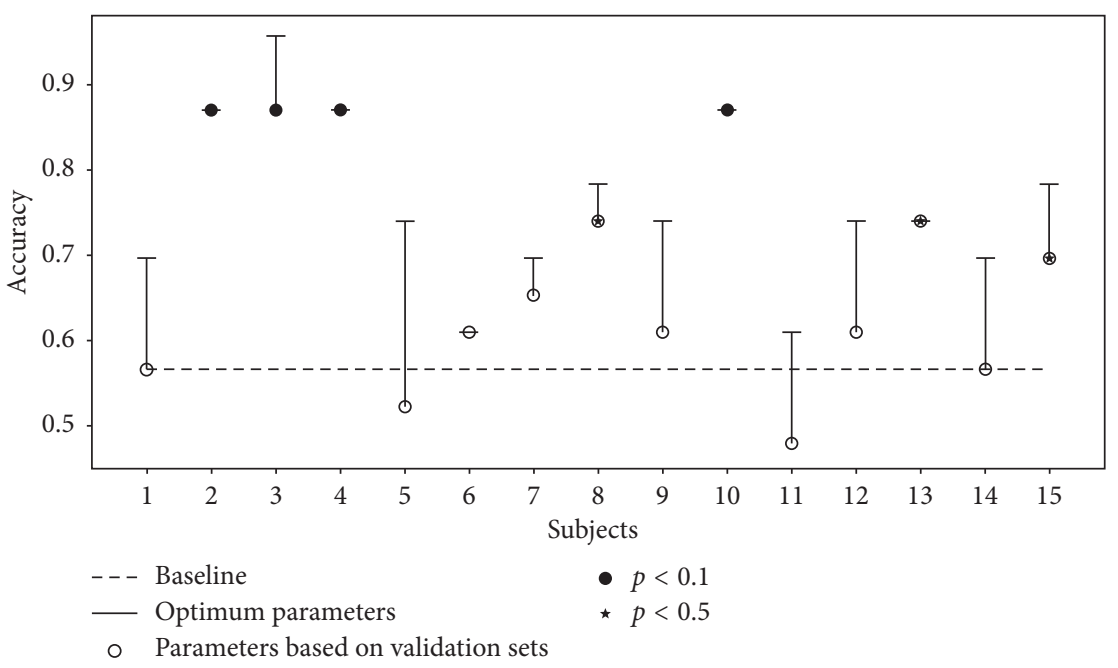

FIgURE 3: Test accuracies for the SVM pseudotrial classifier trained on average categories and tested on average categories. The $x$-axis refers to the hold-out test subject. Chance level prediction accuracy is 0.565 (dashed line). Significance estimated by permutation testing (1000 iterations).

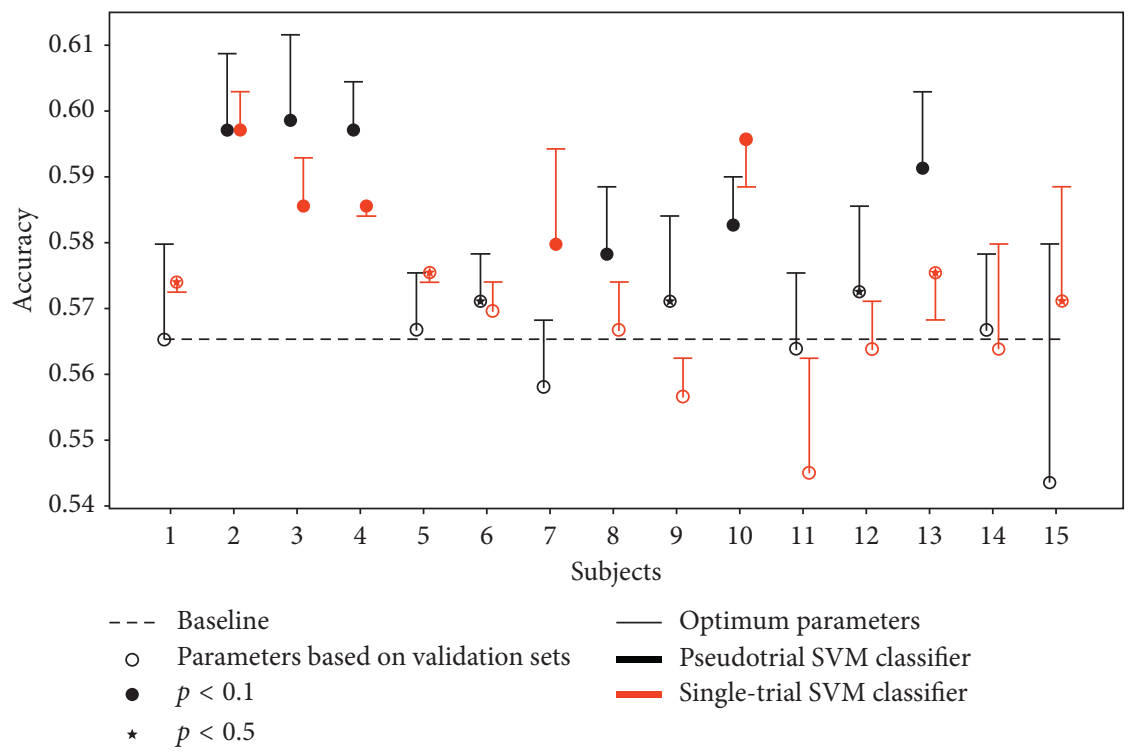

Figure 4: Test accuracies for a classifier trained on pseudotrials (averaged categories) (black) or trained on single-trials (red) and tested on single-trials. The $x$-axis refers to the hold-out test subject. Chance level prediction accuracy is 0.565 (dashed line). Note. In some cases, the "optimum parameters" are not found to be optimum, which can be explained by different training phases of the two single-trial classifiers. The classifier based on validation sets was trained on 13 subjects while the classifier with parameters based on the test set was trained on 14 subjects. For 5 out of 15 subjects, the classifier based on 13 subjects was able to obtain higher accuracies.

pseudotrial classifier exploited a larger proportion of earlier time points compared to the single-trial classifier. The sensitivity maps for the single-trial and pseudotrial classifiers suggest that despite the difference in number and type of trials, the classifiers are similar.

\section{Discussion}

In the current work, we approach the challenges of EEGbased decoding: nonlaboratory settings, user-friendly wireless EEG acquisition equipment with dry electrodes, natural stimuli, no repetition of experimental stimuli trials, and no exclusion of participants. Thus, our work is centered around (1) ecological validity and portability and (2) generalizability. The potential benefits of mitigating these challenges are to study the brain dynamics in natural settings and for applications in real-life scenarios.

Our motivation for working with a portable, dry-electrode EEG system is to increase the EEG usability in terms of affordability, mobility, and ease of maintenance. These factors are crucial in applied contexts in everyday settings, such as the development of real-time EEG neurofeedback systems. It has recently been demonstrated that commercial-grade EEG equipment compares to high-grade equipment in laboratory 


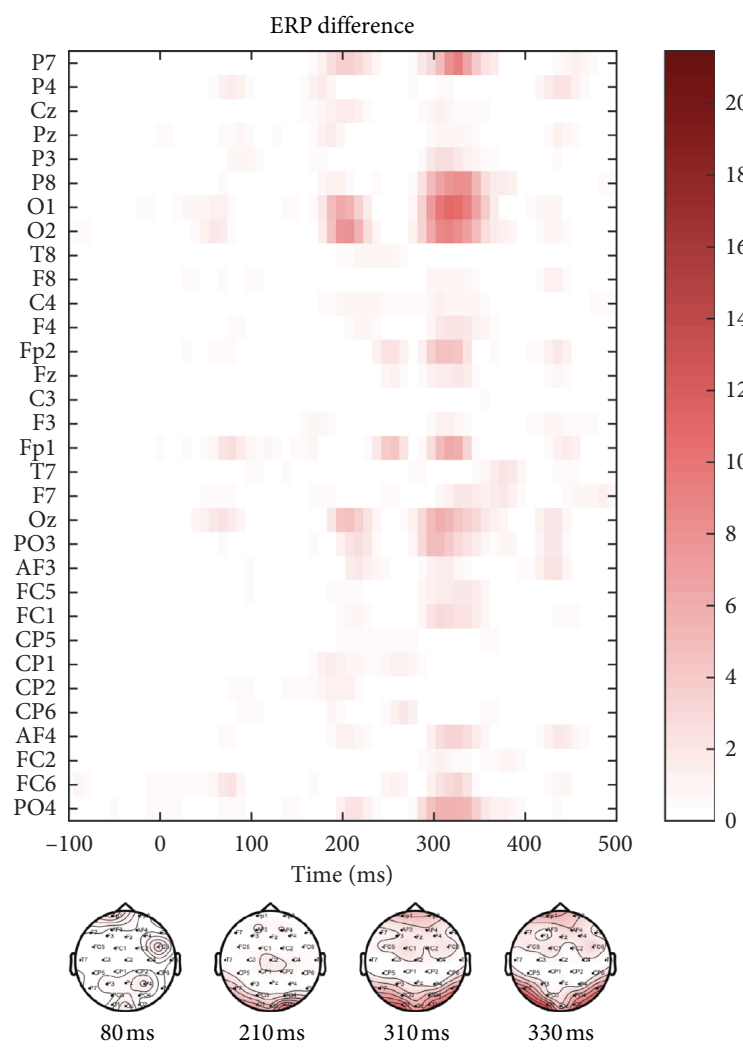

(a)

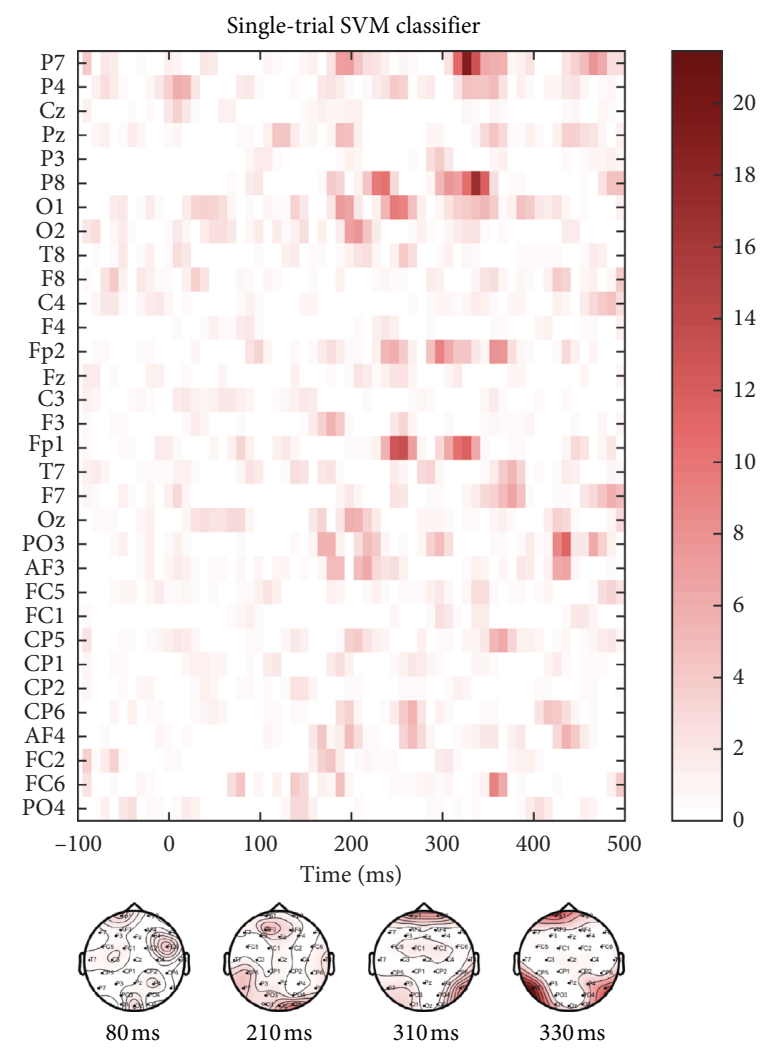

(b)

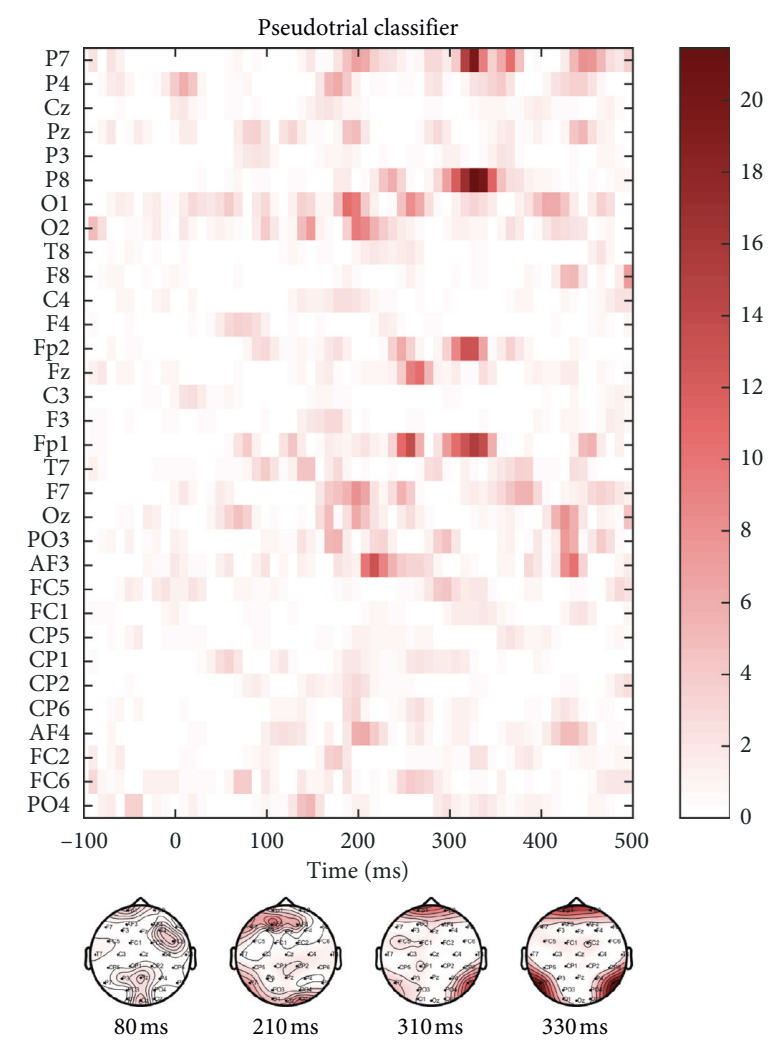

(c)

FIGURE 5: Effect sizes for (a) animate/inanimate ERP difference map and (b, c) the sensitivity maps of the single-trial and pseudotrial SVM classifiers. Effect sizes were computed based on 100 NPAIRS resampling splits. 
settings in terms of neural reliability as quantified by intersubject correlation [34]. Furthermore, a systematic comparison between a wireless dry EEG system and a conventional laboratory-based wet EEG system shows similar performance in terms of signal quality [35].

We aim to increase the generalization ability of our decoding models. To do so, we evaluate decoding ability in an intersubject design, i.e., leave-one-subject-out approach [18]. Prior studies in EEG-based decoding, in particular for BCIs, have focused on building classifiers to decode subjectspecific brain patterns (see [36] for review). Intersubject generalized BCI has the advantage of saving time in BCI sessions, and several research groups have made effort to develop intersubject generalized BCI systems for decoding of motor imagery-related EEG $[37,38]$. Successful intersubject classification requires extraction of globally relevant signal features from each training subject [18]. In the current work, we take a step towards increasing generalizability by building intersubject EEG-based decoding models.

Our ultimate goal is to decode actual semantic differences between natural categories; thus, we perform low-level visual feature standardization of experimental trials prior to the experiment, investigate time dependency of the EEG response throughout the experiment, and perform ASR to reduce this dependency (Section 2.5). Moreover, the stimuli in our experimental paradigm consisted of complex everyday scenes and noniconic views of objects [17]. Animate and inanimate images were similar in composition, i.e., an object or animal in its natural surroundings (Figure 1(a)).

4.1. Data Preprocessing of Temporal Trends. There will naturally be continuous variations in EEG recordings over time. Since our experimental paradigm lasted approximately 40 minutes, we investigated temporal trends in the EEG data (Figures S2 and S3) and perform artifact subspace reconstruction (ASR) [25] to reduce confounding temporal trends in further analyses. The unwanted nonstationarity of the EEG signal arises from electrodes gradually losing or gaining connection to the scalp, an increasing tension of facial muscles or other artifactual currents [39, 40]. If the data are epoched, the drift may misleadingly appear as a pattern reproducible over trials, a tendency that may be further reinforced by component analysis techniques that emphasize repeatable components [41]. Slow linear drifts can be removed by employing high-pass filters; however, more complicated temporal effects are harder to remove. Furthermore, employing high-pass filters may risk introducing new artifacts. As an alternative, recent studies suggest performing robust detrending, where the trend of each channel is determined and then regressed out [41, 42]. We observe that by employing ASR, the time dependency was reduced for most subjects (Figures S2 and S3). However, it would be interesting to investigate more complex detrending algorithms to also make sure that high-pass filtering is not impairing our results.

4.2. Event-Related Potential Analysis. Previous work on visual stimuli decoding demonstrate semantic category specificity at both early $(\sim 150 \mathrm{~ms})$ and late $(\sim 400 \mathrm{~ms})$ intervals of the visually evoked potential $[43,44]$. ERP studies indicate that category-attribute interactions (natural/ nonnatural) emerge as early as $116 \mathrm{~ms}$ after stimulus onset over frontocentral scalp regions and at 150 and $200 \mathrm{~ms}$ after stimulus onset over occipitoparietal scalp regions [45]. Kaneshiro et al. [9] demonstrate that the first $500 \mathrm{~ms}$ of single-trial EEG responses contain information for successful category decoding between human faces and objects and above chance object classification as early as 48-128 ms after stimulus onset [9]. For animate versus inanimate images, ERP differences have been demonstrated detectable within $150 \mathrm{~ms}$ of presentation [46]. However, there appears to be uncertainty whether these early ERP differences represent low-level visual stimuli or actual highlevel differences. We observe the major difference between animate/inanimate ERPs around $210 \mathrm{~ms}$ and $320 \mathrm{~ms}$ (Figures 2 and S4). Akin to our results, Carlson et al. [47] found that high-level categories (animacy) were maximally decodable around $240 \mathrm{~ms}$ from MEG recordings [47]. Lastly, we observe that ERP signatures were highly variable among subjects (comparable to [11]), which challenges the intersubject model generalizability with our sample size of 15 subjects.

4.3. Support Vector Machine Classification. In this study, we adopted RBF kernel SVM classifiers to classify between animate/inanimate natural visual stimuli in a leave-onesubject-out approach. SVM classifiers have previously been implemented for EEG-based decoding. SVM in combination with independent component analysis data processing has been used to classify whether a visual object is present or absent from EEG [12]. Zafar et al. [15] proposed a hybrid algorithm using convolutional neural networks for feature extraction and likelihood-ratio-based score fusion for prediction of brain activity from EEG [15]. TaghizadehSarabi et al. extracted wavelet features from EEG, and selected features are classified using a "one-against-one" SVM multiclass classifier with optimum SVM parameters set separately for each subject [13].

We implemented single-trial and pseudotrial (i.e., averaged categories) SVM classifiers and found very similar performance of the single-trial and pseudotrial classifiers for prediction of single-trial subjects (Figure 4). As the pseudotrial classifier is significantly faster to train, a full-nested cross-validation scheme was feasible. The fact that the two classifiers have similar performance indicates that the reduced sample size in the pseudotrial classifier is offset by the better signal-to-noise ratio of averaged trials. The fast training of the pseudotrial classifier allows for parameter optimization and unbiased performance evaluation.

Based on the leave-one-subject-out classification performance (Figures 3 and 4), it is evident that there is a difference in how well the classifier generalizes across subjects, which partly is due to the diversity of ERP signatures across subjects (Figure S1). For some subjects, low accuracy is caused by a parameter mismatch between trials belonging to that subject and its validation sets. For 
other subjects, the SVM model is not capable of capturing their data even when parameters are based on that subject, due to the poor signal-to-noise level. Furthermore, intersubject generalizability in EEG is complicated by multiple factors. The signal-to-noise ratio at each electrode is affected by the contact to the scalp which is influenced by local differences in skin condition and hair, the spatial location of electrodes relative to underlying cortex will vary according to anatomical head differences, and there may be individual differences in functional localization across participants.

Both SVM classifiers utilized a relatively large number of support vectors. The single-trial SVM classifier used for computing the sensitivity map had model coefficients $\alpha=-1.5, \ldots, 1.5$, where $1204 \alpha$ values out of 10350 were equal to 0 (9146 support vectors). The pseudotrial classifier had model coefficients in the range $\alpha=-7.2, \ldots, 7.2$, and 46 out of 345 coefficients were zero (299 support vectors). The high number of obtained support vectors indicates a poor EEG signal-to-noise ratio and the complexity of the classification problem [26].

4.4. Sensitivity Mapping. In the current work, we ask which parts of the EEG signatures are exploited by the SVM decoding classifiers. We investigated the probabilistic sensitivity map for single-trial and pseudotrial SVM classifiers based on a binary classification task. We identified spatial and temporal regions where discriminative information resides, and found these EEG features comparable to the difference map between raw ERP responses for animate and inanimate trials. We observe the most prominent difference in animate/ inanimate ERPs around $210 \mathrm{~ms}$ and $320 \mathrm{~ms}$ (Figures 2 and S4), and these time points are also exploited by the SVM classifiers to a large extent (Figure 5).

The sensitivity maps for both SVM classifiers reveal that the occipital/parietal channels where visual stimuli are known to be processed $[9,11]$ are major channels of interest in the classification task. Furthermore, we note that Fp1 and Fp2 channels are important in the constructed classifiers (Figure 5). These two frontal channels also display significant differences in animate/inanimate ERPs across all subjects (Figure S4), which might be explained by a difference in eye movements depending on the semantic category. Some studies report that frontal cortex activation is involved in distinguishing between visual stimuli [14], and it has been proposed that frontal activation during visual processing is a result of the attentional and anticipatory state of the subject [48]. However, it is also possible that the frontal channels explain the noise in the informative channels [49].

Based on the similarity between the sensitivity maps for single-trial and pseudotrial classifiers (Figure 5), we conclude that these classifiers exploit the same EEG features to a large extent. We therefore investigated whether the pseudotrial classifier is able to predict on single-trial test subjects. We demonstrate that classifiers trained on averaged pseudotrials perform on par with classifiers trained on a large set of noisy single-trial samples (Figure 4).

\section{Conclusion}

We investigate scalp EEG recorded with portable 32 dryelectrode EEG equipment from healthy subjects under natural stimuli. We accomplish unbiased decoding of single-trial EEG using SVM models trained on denoised (averaged) pseudotrials, thus facilitating fast classifier training, parameter optimization, and unbiased performance evaluation. The SVM classifiers were evaluated in a intersubject approach, thus probing generalizability across participants. We propose a novel methodology for evaluating and computing sensitivity maps for EEG-based SVM classifiers, allowing for visualization of discriminative SVM classifier information. We implement an NPAIRS resampling scheme to compute sensitivity map effect sizes and demonstrate high similarity between sensitivity map effect sizes of classifiers trained on small samples of denoised, averaged data (pseudotrial) and large samples of noisy data (single-trial). Finally, by linking temporal and spatial features of EEG to training of SVM classifiers, we take an essential step in understanding how machine learning techniques exploit neural signals.

\section{Appendix}

\section{A. Manual Exclusion Criteria for Image Selection}

Manual exclusion criteria for MS COCO images [17] for the experimental paradigm:

(i) Object unidentifiable

(ii) Object not correctly categorized

(iii) Different object profoundly more in focus

(iv) Color scale manipulation

(v) Frame or text overlay on image

(vi) Distorted photograph angle

(vii) Inappropriate image

\section{B. Sensitivity Map Python Pseudocode}

The following piece of pseudocode shows how to compute the sensitivity map for an SVM classifier with an RBF kernel across all trials using Python and NumPy (np).

$$
\begin{aligned}
& \text { map }=\operatorname{np} \cdot \operatorname{matmul}(X, \text { np.matmul(np.diag(alpha), } k))- \\
& (\text { np.matmul( } X \text {, (np.diag(np.matmul(alpha, } k))))) \\
& s=\operatorname{np} \cdot \operatorname{sum}(\text { np.square }(\text { map), axis }=1) / \text { np.size (alpha) }
\end{aligned}
$$

$k$ denotes the $(N \times N)$ RBF training kernel matrix from equation (2), with $N$ as the number of training examples. Alpha denotes a $(1 \times N)$ vector with model coefficients. $X$ denotes a $(P \times N)$ matrix with training examples in columns. $s$ is a $(P \times 1)$ vector with estimates of channel sensitivities for each time point, which can be resized into a matrix of size (number of channels, number of time points) for EEG-based sensitivity map visualization. 


\section{Data Availability}

The code is available at the following link: https://github. com/gretatuckute/DecodingSensitivityMapping.

\section{Conflicts of Interest}

The authors declare that they have no conflicts of interest regarding the publication of this paper.

\section{Authors' Contributions}

G.T., N.P., and L.K.H. designed research; G.T. and N.P. acquired data; D.S., G.T., and N.P. performed initial data analyses; G.T., S.T.H., and L.K.H. performed research; and G.T., S.T.H., and L.K.H. wrote the paper.

\section{Acknowledgments}

This work was supported by the Novo Nordisk Foundation Interdisciplinary Synergy Program 2014 (Biophysically Adjusted State-Informed Cortex Stimulation (BASICS)) (NNF14OC0011413).

\section{Supplementary Materials}

Supplementary 1. Image IDs, supercategories, and categories for all images used in the experiment from the Microsoft Common Objects in Context (MS COCO) image database.

Supplementary 2. Figures S1-S10 contain supplementary material and are used for reference in the main manuscript.

\section{References}

[1] C. Gerlach, "A review of functional imaging studies on category specificity," Journal of Cognitive Neuroscience, vol. 19, no. 2, pp. 296-314, 2007.

[2] J.-D. Haynes and G. Rees, "Decoding mental states from brain activity in humans," Nature Reviews Neuroscience, vol. 7, no. 7, pp. 523-534, 2006.

[3] U. Güçlü and M. A. J. van Gerven, "Increasingly complex representations of natural movies across the dorsal stream are shared between subjects," NeuroImage, vol. 145, pp. 329-336, 2017.

[4] A. G. Huth, T. Lee, S. Nishimoto, N. Y. Bilenko, A. T. Vu, and J. L. Gallant, "Decoding the semantic content of natural movies from human brain activity," Frontiers in Systems Neuroscience, vol. 10, pp. 1-16, 2016.

[5] A. G. Huth, S. Nishimoto, A. T. Vu, and J. L. Gallant, "A continuous semantic space describes the representation of thousands of object and action categories across the human brain," Neuron, vol. 76, no. 6, pp. 1210-1224, 2012.

[6] K. N. Kay, T. Naselaris, R. J. Prenger, and J. L. Gallant, "Identifying natural images from human brain activity," Nature, vol. 452, no. 7185, pp. 352-355, 2008.

[7] S. Nishimoto, A. T. Vu, T. Naselaris, Y. Benjamini, B. Yu, and J. L. Gallant, "Reconstructing visual experiences from brain activity evoked by natural movies," Current Biology, vol. 21, no. 19 , pp. 1641-1646, 2011.

[8] R. J. Prenger, J. L. Gallant, K. N. Kay, T. Naselaris, and M. Oliver, "Bayesian reconstruction of natural images from human brain activity," Neuron, vol. 63, no. 6, pp. 902-915, 2009.

[9] B. Kaneshiro, M. Perreau Guimaraes, H.-S. Kim, A. M. Norcia, and P. Suppes, "A representational similarity analysis of the dynamics of object processing using single-trial EEG classification," PLoS One, vol. 10, no. 8, Article ID e0135697, 2015.

[10] B. Murphy, M. Poesio, F. Bovolo, L. Bruzzone, M. Dalponte, and H. Lakany, "EEG decoding of semantic category reveals distributed representations for single concepts," Brain and Language, vol. 117, no. 1, pp. 12-22, 2011.

[11] I. Simanova, M. van Gerven, R. Oostenveld, and P. Hagoort, "Identifying object categories from event-related EEG: toward decoding of conceptual representations," PLoS One, vol. 5, no. 12, Article ID e14465, 2010.

[12] A. X. Stewart, A. Nuthmann, and G. Sanguinetti, "Single-trial classification of EEG in a visual object task using ICA and machine learning," Journal of Neuroscience Methods, vol. 228, pp. 1-14, 2014.

[13] M. Taghizadeh-Sarabi, M. R. Daliri, and K. S. Niksirat, "Decoding objects of basic categories from electroencephalographic signals using wavelet transform and support vector machines," Brain Topography, vol. 28, no. 1, pp. 33-46, 2015.

[14] C. Wang, S. Xiong, X. Hu, L. Yao, and J. Zhang, "Combining features from ERP components in single-trial EEG for discriminating four-category visual objects," Journal of Neural Engineering, vol. 9, no. 5, article 056013, 2012.

[15] R. Zafar, S. C. Dass, and A. S. Malik, "Electroencephalogrambased decoding cognitive states using convolutional neural network and likelihood ratio based score fusion," PLoS One, vol. 12, no. 5, Article ID e0178410, 23 pages, 2017.

[16] L. F. Nicolas-Alonso and J. Gomez-Gil, "Brain computer interfaces, a review," Sensors, vol. 12, no. 2, pp. 1211-1279, 2012.

[17] T. Y. Lin, M. Maire, S. Belongie et al., "Microsoft COCO: common objects in context," in Computer Vision-ECCV 2014, pp. 740-755, Springer, Berlin, Germany, 2014.

[18] U. Kjems, L. K. Hansen, J. Anderson et al., "The quantitative evaluation of functional neuroimaging experiments: mutual information learning curves," NeuroImage, vol. 15, no. 4, pp. 772-786, 2002.

[19] R. S. Andersen, A. U. Eliasen, N. Pedersen, M. R. Andersen, S. T. Hansen, and L. K. Hansen, "EEG source imaging assists decoding in a face recognition task," http://arxiv.org/abs/ 1704.05748, 2017.

[20] F. Lotte, M. Congedo, A. Lécuyer, F. Lamarche, and B. Arnaldi, "A review of classification algorithms for EEGbased brain-computer interfaces," Journal of Neural Engineering, vol. 4, no. 2, pp. R1-R13, 2007.

[21] P. M. Rasmussen, K. H. Madsen, T. E. Lund, and L. K. Hansen, "Visualization of nonlinear kernel models in neuroimaging by sensitivity maps," NeuroImage, vol. 55, no. 3, pp. 1120-1131, 2011.

[22] S. C. Strother, J. Anderson, L. K. Hansen et al., "The quantitative evaluation of functional neuroimaging experiments: the NPAIRS data analysis framework," NeuroImage, vol. 15, no. 4, pp. 747-771, 2002.

[23] Den Nationale Videnskabsetiske Komit', Vejledning Om Anmeldelse, Indberetning $M v$, (Sundhedsvidenskablige Forskningsprojekter), Den Nationale Videnskabsetiske Komit', København, Denmark, 2014.

[24] J. W. Peirce, "Generating stimuli for neuroscience using PsychoPy," Frontiers in Neuroinformatics, vol. 2, p. 10, 2009. 
[25] T. R. Mullen, C. A. E. Kothe, Y. M. Chi et al., "Real-time neuroimaging and cognitive monitoring using wearable dry EEG," IEEE Transactions on Biomedical Engineering, vol. 62, no. 11, pp. 2553-2567, 2015.

[26] C. Cortes and V. Vapnik, "Support-vector networks," Machine Learning, vol. 20, no. 3, pp. 273-279, 1995.

[27] B. Lautrup, L. K. Hansen, I. Law, N. Mörch, C. Svarer, and S. C. Strother, "Massive weight sharing: a cure for extremely ill-posed problems," in Proceedings of the Workshop on Supercomputing in Brain Research: From Tomography to Neural Networks, HLRZ, Jülich, Germany, November 1994.

[28] M. Guggenmos, P. Sterzer, and R. M. Cichy, "Multivariate pattern analysis for MEG: a comparison of dissimilarity measures," NeuroImage, vol. 173, pp. 434-447, 2018.

[29] J. Polich, "Updating P300: an integrative theory of P3a and P3b," Clinical Neurophysiology, vol. 118, no. 10, pp. 21282148, 2007.

[30] R. M. Cichy, D. Pantazis, and A. Oliva, "Resolving human object recognition in space and time," Nature Neuroscience, vol. 17, no. 3, pp. 455-462, 2014.

[31] F. Pedregosa, G. Varoquaux, A. Gramfort et al., "Scikit-learn: machine learning in Python," Journal of Machine Learning Research, vol. 12, pp. 2825-2830, 2011.

[32] C. M. Bishop, Pattern Recognition and Machine Learning (Information Science and Statistics), Springer-Verlag, Berlin, Heidelberg, Germany, 2006.

[33] B. Efron and R. J. Tibshirani, An Introduction to the Bootstrap, CRC Press, Boca Raton, FL, USA, 1994.

[34] A. T. Poulsen, S. Kamronn, J. Dmochowski, L. C. Parra, and L. K. Hansen, "EEG in the classroom: synchronised neural recordings during video presentation," Scientific Reports, vol. 7, pp. 1-9, 2017.

[35] J. W. Y. Kam, S. Griffin, A. Shen et al., "Systematic comparison between a wireless EEG system with dry electrodes and a wired EEG system with wet electrodes," NeuroImage, vol. 184, pp. 119-129, 2019.

[36] H. Cecotti, "Spelling with non-invasive brain-computer interfaces - current and future trends," Journal of Physiology Paris, vol. 105, no. 1-3, pp. 106-114, 2011.

[37] H.-L. Halme and L. Parkkonen, "Across-subject offline decoding of motor imagery from MEG and EEG," Scientific Reports, pp. 1-12, 2018.

[38] A. M. Ray, R. Sitaram, M. Rana et al., "A subject-independent pattern-based brain-computer interface," Frontiers in Behavioral Neuroscience, vol. 9, p. 269, 2015.

[39] A. Delorme, T. Sejnowski, and S. Makeig, "Enhanced detection of artifacts in EEG data using higher-order statistics and independent component analysis," NeuroImage, vol. 34, no. 4, pp. 1443-1449, 2007.

[40] A. J. Rowan and E. Tolunsky, A Primer of EEG: With a MiniAtlas, Butterworth-Heinemann Medical, Oxford, UK, 2003.

[41] A. de Cheveigné and D. Arzounian, "Robust detrending, rereferencing, outlier detection, and inpainting for multichannel data," NeuroImage, vol. 172, pp. 903-912, 2018.

[42] J. V. Driel, C. N. L. Olivers, and J. J. Fahrenfort, High-Pass Filtering Artifacts in Multivariate Classification of Neural Time Series Data, bioRxiv, Cold Spring Harbor, NY, USA, 2019.

[43] G. A. Rousselet, J. S. Husk, P. J. Bennett, and A. B. Sekuler, "Single-trial EEG dynamics of object and face visual processing," NeuroImage, vol. 36, no. 3, pp. 843-862, 2007.

[44] G. A. Rousselet, M. J.-M. Mace, and M. Fabre-Thorpe, "Animal and human faces in natural scenes: how specific to human faces is the N170 ERP component?," Journal of Vision, vol. 4, no. 1, p. 2, 2004.
[45] K. Hoenig, E.-J. Sim, V. Bochev, B. Herrnberger, and M. Kiefer, "Conceptual flexibility in the human brain: dynamic recruitment of semantic maps from visual, motor, and motion-related areas," Journal of Cognitive Neuroscience, vol. 20, no. 10, pp. 1799-1814, 2008.

[46] S. Thorpe, D. Fize, and C. Marlot, "Speed of processing in the human visual system," Nature, vol. 381, no. 6582, pp. 520-522, 1996.

[47] T. Carlson, D. A. Tovar, A. Alink, and N. Kriegeskorte, "Representational dynamics of object vision: the first 1000 ms," Journal of Vision, vol. 13, no. 10, p. 1, 2013.

[48] J. Foxe and G. Simpson, "Flow of activation from V1 to frontal cortex in humans," Experimental Brain Research, vol. 142, no. 1, pp. 139-150, 2002.

[49] B. Blankertz, S. Lemm, M. Treder, S. Haufe, and K.-R. Müller, "Single-trial analysis and classification of ERP components - a tutorial,” NeuroImage, vol. 56, no. 2, pp. 814-825, 2011. 


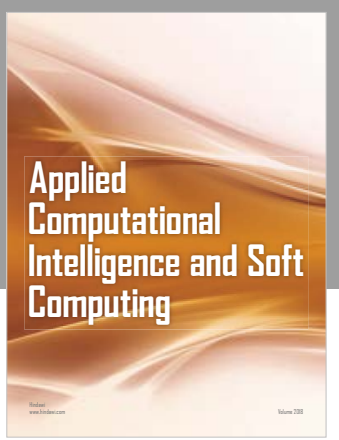

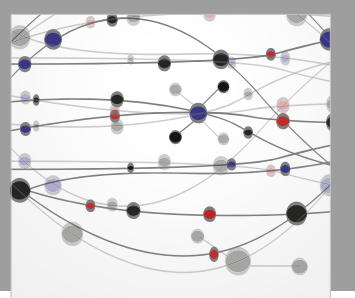

The Scientific World Journal
Submit your manuscripts at

Computing
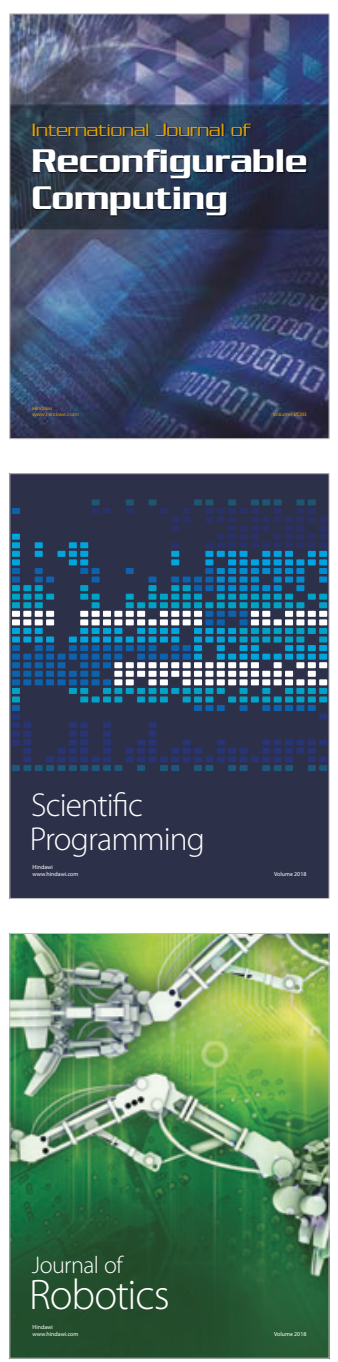

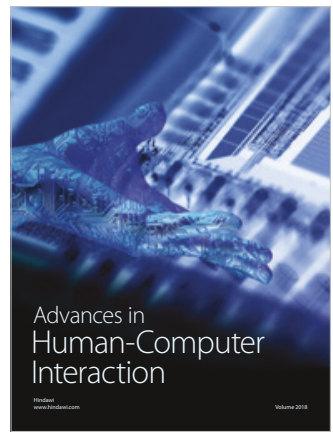

Human-Compute

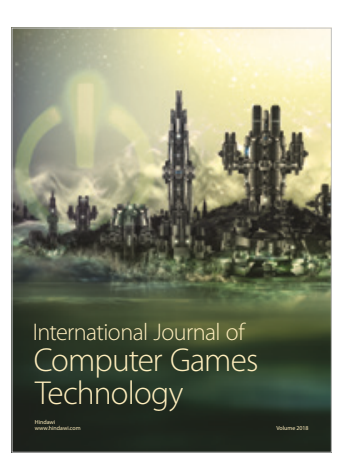

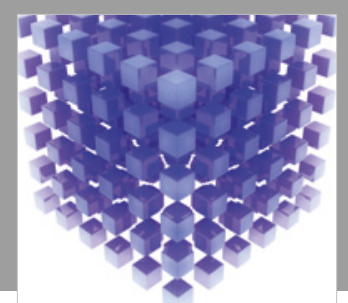

Mathematical Problems in Engineering

\section{Engincering}
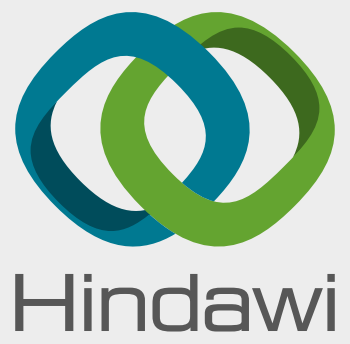

www.hindawi.com
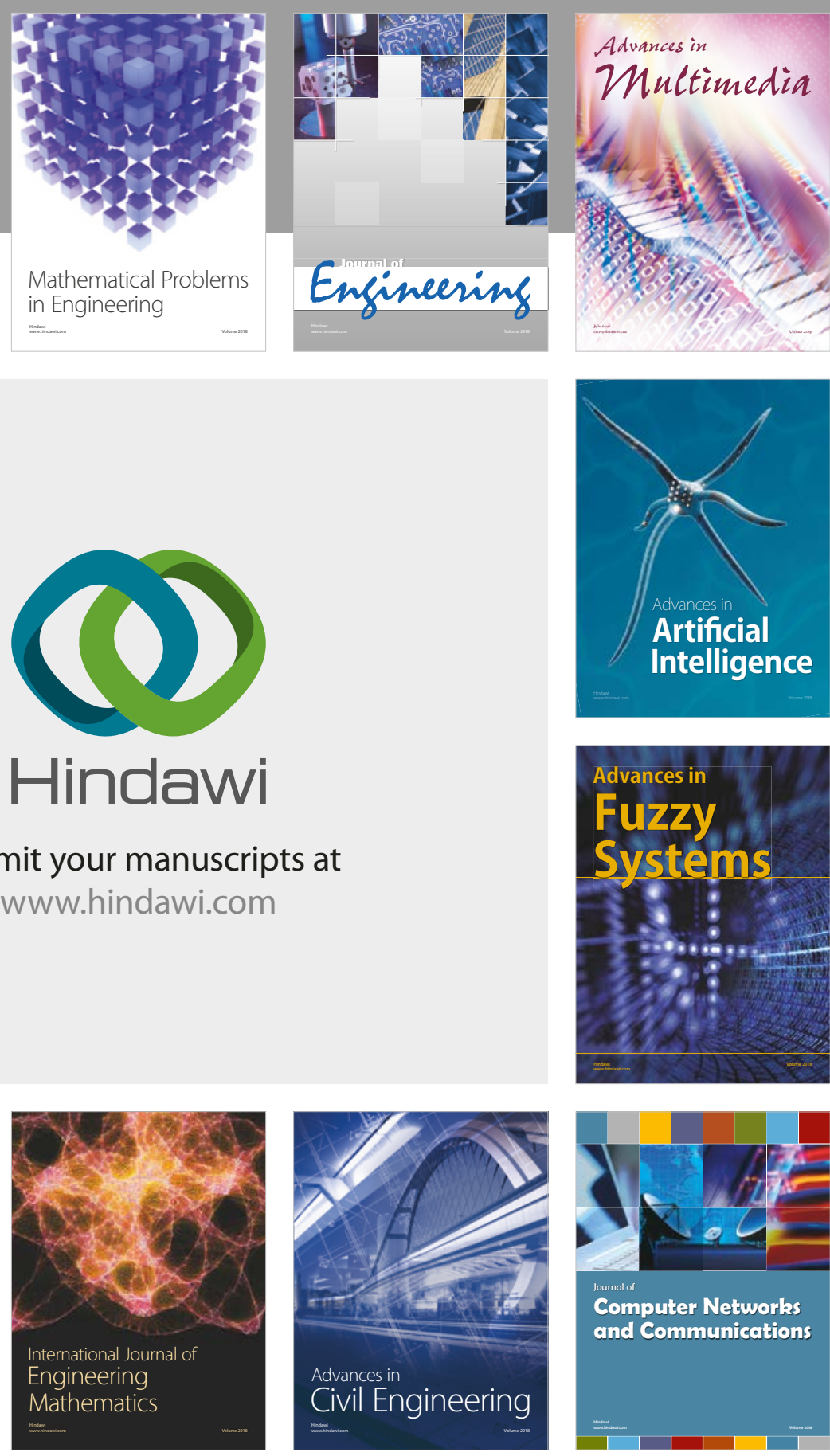

Computer Networks and Communications

Multimedia
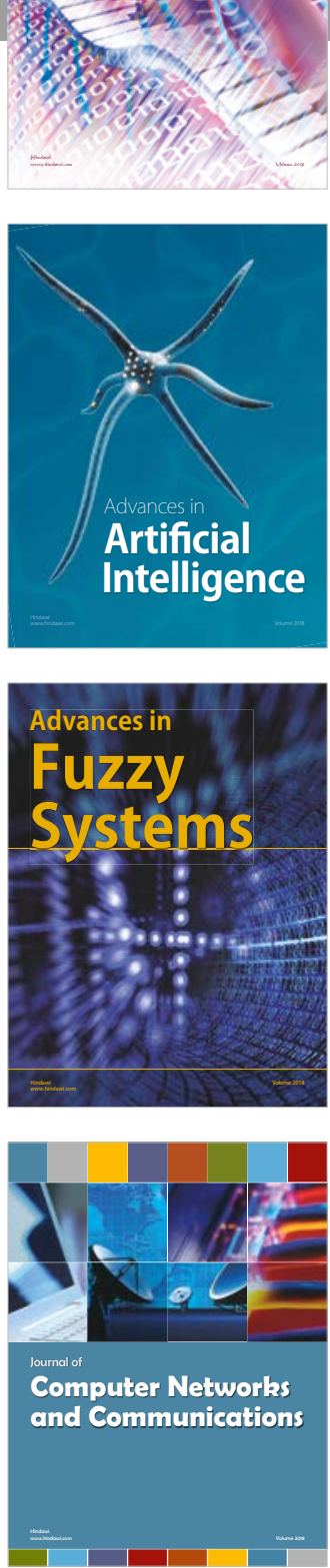

Advances in

Modelling \&

Simulation

in Engineering

interaction

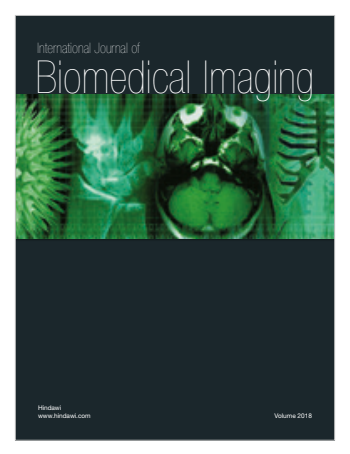

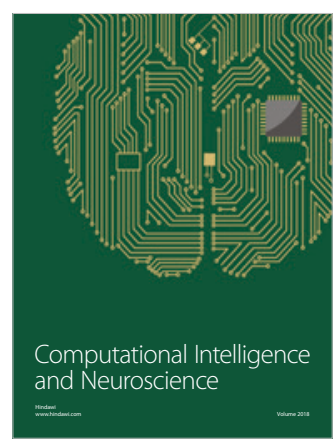

